Journal of Clinical Investigation

Iol. $41, \mathrm{No} .7,1962$

\title{
MEASUREMENT OF THE CEPHALIC AND CEREBRAL BLOOD FLOW FRACTIONS OF THE CARDIAC OUTPUT IN MAN *
}

\author{
BY LEO A. SAPIRSTEIN
}

(From the Department of Physiology, The Ohio State University, Columbus, Ohio)

(Submitted for publication January 8, 1962; accepted February 17, 1962)

The present report is concerned with the application of the indicator fractionation technique (1) to the measurement of the cephalic and cerebral blood flow fractions of the cardiac output in man. The indicator fractionation technique was originally developed to permit the determination of regional blood flow in experimental animals (1, 2 ). The blood flow measurement was made by determining the pattern of distribution of a single mass of injected indicator at a time when the indicator had been delivered to the organs by their arterial supply but presumably had not yet left any of the organs by way of their venous drainage to a significant extent. At such time the distribution of the indicator can be taken to correspond to the distribution of the cardiac output if the arterial blood supplying all organs is homogeneous.

In order to characterize the situation existing at this time (just after arterial delivery and before venous drainage) a group of observations is made of organ indicator content at various times after indicator injection. These observations, extrapolated to zero time (assumed to be the moment of arterial delivery), describe the distribution of indicator before significant venous losses have occurred. Since organ indicator content is measured on excised organs after the animals have been killed, the observations from which the extrapolation is made are based on several animals

* Reported in part to the 1961 Fall Meetings of the American Physiological Society, Bloomington, Ind. Most of this material was previously presented in a progress report dated May 15, 1960, to the Aerospace Medical Laboratory, Wright-Patterson Air Force Base, Ohio. This work was supported by a grant-in-aid from the Central Ohio Heart Association and a grant-in-aid from the National Heart Institute. Some of this work was carried out in partial fulfillment of a contract between The Ohio State University Research Foundation and The Acceleration Section, Biophysics Branch, Aerospace Medical Laboratory, Wright-Patterson Air Force Base, Ohio. taken from a homogeneous group killed at different times after administration of the indicator.

The use of $\gamma$-emitting isotopes as indicators, in conjunction with the development of large wellcounters that can incorporate a human extremity, has made it possible to apply the same principle to the measurement of blood flow in the human hand (3). The indicator content is measured by external counting of the extremity introduced into the well; continuous monitoring of the extremity count in a single individual does away with the necessity for using groups in characterizing the time course of organ indicator content. Continuous monitoring has the further advantage that the uncertainties of extrapolation from later data are diminished. Although any indicator is theoretically suitable in this technique, difficulties in extrapolation (see below) dictate the use of certain ones for particular measurements.

In the case of most organs, either potassium ${ }^{42}$ or rubidium $^{86}$ is a suitable indicator. Neither label is suitable for the measurement of cerebral blood flow in animals. The latter can be conveniently determined by the use of iodo ${ }^{131}$-antipyrine $\left(\mathrm{I}^{131}\right)$ as the indicator (4).

As will be shown below, $\mathrm{I}^{131}$ appears to be suitable for the determination of the cephalic blood flow fraction in man. $\mathrm{K}^{42}$ appears, with some limitations, to be suitable for the measurement of the noncerebral cephalic blood flow fraction. The cerebral blood flow fraction should, therefore, be measurable as the difference between these two values.

\section{THEORETICAL CONSIDERATIONS}

The theoretical basis for the indicator fractionation technique has been described in several publications from this laboratory (1-6). Basically, the technique depends on the fact that any indicator having the same extraction ratio in an organ and in the whole body will be distributed between the organ and the body in the same manner as is the blood supply.

At the present time the requirement of identical extrac- 
tion ratios is not generally met by any practical indicator. Glass microspheres appear to meet this need but present certain technical problems; however, it is possible to describe circumstances in which the requirement is met by many indicators. These circumstances are those in which the extraction ratio is 1.00 for both organ and body (complete removal of indicator).

For any nonexcreted, nonmetabolized indicator, the extraction ratio (defined as the ratio of the integrated arteriovenous concentration difference to the integrated arterial concentration-both taken from the moment of indicator administration) will have a value of 1.00 across any vascular bed for a short time after its first introduction into the blood stream. This is a consequence of the fact that there is a finite transit time for the passage of indicator from arterial to venous blood; during this time the arterial concentration integral exists, while the venous concentration integral is zero. Gradually, as the indicator makes its appearance in the venous blood, the extraction ratio will diminish, eventually approaching zero in every region.

It is not to be expected that the transition from the initial state (extraction ratio 1.00) to the final state will occur at the same rate in every vascular bed. In some the transition will be very rapid; in others it may be slow. Clearly, those areas in which the transition is rapid will have a lesser extraction ratio than have those areas in which the transition is slow; they will lose indicator with time, whereas the areas in which the transition is slow will gain it continuously. During the transition the distribution pattern of the indicator will be modified from one that is dominated by the pattern of volume flow to one that is dominated by the pattern of volume of distribution. The uncontaminated, flow-dominated situation can be visualized only when all extraction ratios are identical, i.e., at the moment of arterial delivery.

The examination of the moment of arterial delivery is most effectively accomplished by observation of the early time course of indicator shifts into or out of organs, with extrapolation of the observations to zero time. Two circumstances stand in the way of carrying out this extrapolation accurately. The first of these has to do with the fact that the transition may be so rapid that it is nearly complete before the first observation can be made. This is what is observed in the rat brain when $\mathrm{K}^{42}$ or $\mathrm{Rb}^{88}$ is the indicator, and the first observation is made 5 seconds after the administration (4). The second circumstance has to do with the fact that there is no single time of arterial delivery (i.e., that the "zero time" to which extrapolation is to be made is variable from organ to organ because of the difference in arterial transit times).

Both of these circumstances are all but eliminated when the entire time course of the indicator content of an organ is exposed to view, and when the organ is found to change its content slowly with time. The extrapolation to the uncertain zero time is quite safe if the dependence of organ content of indicator upon time is a weak one. For example, if an organ changes its indicator content from 20 to 15 per cent of the administered dose in the interval between 10 and 60 seconds after indicator administration, the change per second ( 0.1 per cent per second) extrapolated linearly over the entire 10 -second period preceding the first observation is only 1 per cent; the maximal error in the estimate of the zero time content of the organ would be 5 per cent of the actual value.

The exposure of the entire time course of organ indicator content diminishes considerably the possibility that very early rapid changes may be concealed. The possibility is not altogether eliminated in practice, since the time constant of the early change may be so much less than that of the instrument used that it may be buried in instrument lag.

From these considerations it becomes apparent that the indicator fractionation technique can be applied to the measurement of regional blood flow whenever it is the case that, the early time course of organ indicator content being visualized, the change with time is sufficiently gradual to permit a confident extrapolation to zero time. A moment's consideration of the factors likely to lead to such a gradual transition may prove useful.

Rapid transition of the extraction ratio from 1.00 to nearly zero is the expected result whenever the venous concentration curve preserves the characteristics of the arterial curve. This will occur when the indicator, in passing through the organ, encounters a reservoir that is small in relation to the blood flow rate. On the contrary, the venous concentration values will be small in relation to the arterial, and the extraction ratio will remain high for a long period if the reservoir into which the indicator is emptied is a large one. In effect, by choice of an indicator that has a large volume of distribution everywhere, one is able to prolong considerably the period during which the extraction ratio remains high. When it is possible to find such an indicator, the time during which the pattern of distribution is dominated by flow, rather than by volume, is prolonged.

This is not to say that slow redistribution indicates that there are no differences in the "affinity" of the several organs for the indicator. Affinity may vary many times without significant redistribution. For example, a given organ may have an affinity 1,000 times as great as that of another. At first sight this suggests that it would accumulate 1,000 times as much indicator, and this is in fact true if sufficient time is allowed for the indicator to come to equilibrium. But if the affinity of the second organ for the indicator is such that during the first minute it extracts 95 per cent of the indicator delivered to it by the arterial blood, the first organ can extract no more than 99.995 per cent. There is, indeed, a 1,000-fold difference in affinity; but there is only a 5 per cent difference in the extraction ratio. If these organs had the same blood flow they would contain almost precisely the same amount of indicator after 1 minute, despite their thousandfold difference in "affinity." Only after many recirculations would the affinity difference be displayed.

We may now consider the behavior of a nonhomogeneous organ, consisting of two parts, one of which has a high, the other a very low, affinity for the indicator employed. The indicator content for such an organ can be described as the sum of the indicator content of its two 
parts. The part with a high affinity (assumed that the remainder of the body also has a high affinity for the indicator) will show a gradual change with time, the indicator content increasing with time if the body has a lower affinity, decreasing with time if the body has a higher one. The extrapolation to zero time of the indicator content of this part will describe its fractional blood flow.

The part with the very low affinity will nevertheless have an initial extraction ratio of 1.00 . (It will be recalled that the extraction ratio is defined as the integrated $\mathrm{A}-\mathrm{V} / \mathrm{A}$ from zero time, and that if the time is sufficiently short, the integrated $\mathrm{V}$ is zero.) After a few moments, however, the gross disparity between the two affinities will become manifest, displaying itself as a precipitous decline in the indicator content of this part of the organ. The reader should note that, in this case, the difference between a small and a large affinity is referred to; in the discussion above, the difference between two very large affinities was under consideration. The decline (representing the rapid approach of the extraction ratio to zero) will be so rapid that the part may become quite devoid of indicator within seconds, except for the indicator content of the blood.

The organ as a whole, being the sum of these two parts, will display a high early indicator content. Very rapidly this value will decline to a more stable value, which characterizes the blood flow of the part with high affinity. The early peak and decline, characteristic of the part with low affinity, cannot be used to quantitate its blood flow (see above on the difficulties of extrapolating rapidly changing organ values to zero time). It can, however, be used as evidence of the existence of such a part in the organ studied.

The situation described above is characteristic of the behavior of the rat head toward radiopotassium and radiorubidium. After the injection of either label a small but definite peak can be seen between 3 and 5 seconds. This peak, superimposed on a high, stable value, can be shown to result from the momentary accumulation and washout by the brain of the alkali metal isotopes, the remainder of the head behaving like most organs of the rat in that the initial uptake of the label shows exceedingly slow changes for the next 3 minutes.

From this it appears that the noncephalic cerebral blood flow can be estimated from values obtained with alkali metal isotopes by extrapolating to zero time the slowly changing portion of the head indicator content-time curve. By using another label for which no portion of the head shows a very low extraction ratio-e.g. $\mathrm{I}^{131}$ - so that a slow transition of indicator content from its initial condition is obtained, the entire cephalic blood flow fraction is measurable. The difference between these values should yield the cerebral blood flow.

As will be shown later, the human head behaves qualitatively toward both the alkali metal isotopes and $\mathrm{I}^{181}$ like the rat head. There is, however, an important quantitative difference associated with the much larger relative mass of the human brain. The effect of this difference is to make the value obtained with the alkali metal isotope (the noncerebral cephalic blood flow) higher than it ought to be. The cerebral blood flow obtained by difference is correspondingly low. The nature of the error and its correction will be considered in the Discussion.

\section{MATERIALS AND METHODS}

Counting equipment. The counter used in these experiments was a well-counter large enough to accommodate the entire head. This counter ${ }^{1}$ consists of an array of bismuth tubes in a common gas envelope. The tubes are so arranged that essentially constant geometry is achieved for the head within the well regardless of the exact relationship of the head to the wall and floor of the well. The geometry of the well is such that objects in its longitudinal axis and not enclosed by the walls (such as the thoracic and abdominal organs) are counted with extremely low efficiency. Thus, without special shielding, the counts recorded from a subject whose head is within the well are almost entirely due to the cephalic content of $\boldsymbol{\gamma}$-emitting isotopes.

The counter was used without any shielding in these experiments. Although this resulted in a high background (about 10,000 cpm) the increase in head count was sufficiently great with the amounts of isotope used to permit accurate evaluation of the isotope content of the head. In this connection, a remarkable and as yet unexplained feature of the behavior of this equipment should be noted. The count rate of a sample of either $\mathrm{K}^{42}$ or $\mathrm{I}^{131}$ within the well is independent not only of the position of the sample but also of the volume in which the sample is contained. For example, a sample counting $34,000 \mathrm{cpm}$ in a volume of $10 \mathrm{ml}$ gave precisely the same count in a volume of $4,000 \mathrm{ml}$. For this reason no attempt was made to correct the values obtained for the size of the head.

The count rate was displayed through a Nuclear Chicago rate meter, which recorded through a Texas Instrument Rectiriter. The time constant of the rate meter was set at 0.5 second.

Radioisotopes and standards. The indicators used were $\mathrm{K}^{42} \mathrm{Cl}(\mathrm{P})$ obtained from the Oak Ridge National Laboratory and iodo ${ }^{131}$-antipyrine from Abbott Laboratories. The $\mathrm{K}^{42}$ was injected at a dose of 10 to $20 \mu \mathrm{c}$, the $\mathrm{I}^{131}$ at a dose of 3 to $5 \mu \mathrm{c}$.

The standard solutions contained half the injected dose of either isotope in a volume of $4 \mathrm{~L}$, in a bottle roughly the size of the human head and placed in the well in the same manner as the head of the subject.

The deflection of the recording apparatus being linear, all counts were measured as the elevation of the record above background level; the fraction of the injected dose being measured was obtained by dividing this value by twice the deflection of the 50 per cent standard above background.

Subjects. The experimental subjects were paid volunteers of both sexes. Their ages ranged from 19 to 70 .

1 Constructed to the author's specifications by Dr. Benjamin Schloss of The Nucleonic Corporation of America, 192 DeGraw Street, Brooklyn, N. Y. 


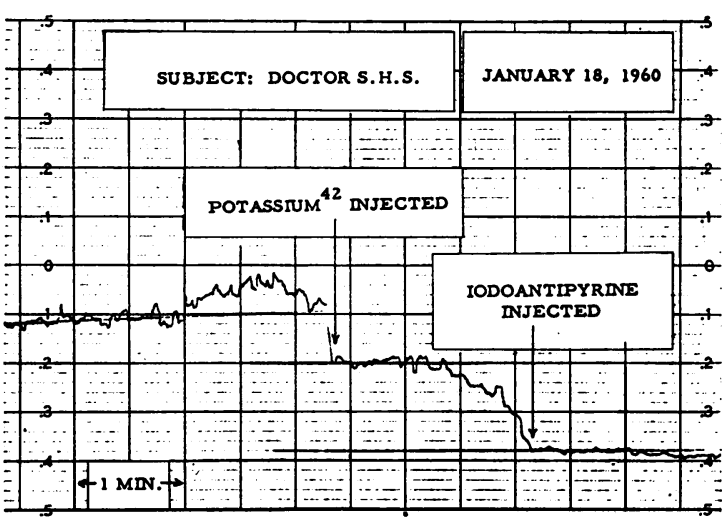

Fig. 1. A tYPICAL RECORD OF THE TRACING OF ${ }^{131}$ AND $\mathrm{K}^{42}$ CONTENT OF THE HUMAN HEAD AFTER INTRAVENOUS INJECTION. Note the relatively constant value reached after $\mathrm{I}^{131}$. The peaking and decline after $\mathrm{K}^{42}$ are characteristic. The extrapolated value for the $\mathrm{K}^{42}$ is based on the behavior of the curve after the first minute.

All were in good health. Some were medical students, some employees of the Physiology Department; two were medical officers of the U. S. Air Force. All subjects were carefully instructed concerning the purpose and the hazards of the contemplated procedure; none who displayed unusual concern was accepted. No attempt was made to keep any of the subjects in basal circumstances.

The subject reclined on a hospital bed with his head enclosed by the well and from 5 to 10 minutes was allowed for him to become used to the situation. During this period a background count was recorded. An injection of one or the other indicator, contained in a volume of $10 \mathrm{ml}$, was then made into an antecubital vein. The count rate from the head was continuously recorded for 2 minutes. By this time, whichever label was used, the count rate was changing very slowly. A second injection of the other label was then made through the same needle, and the count again recorded for 2 minutes.

The label content of the head at the time of injection was determined by comparing the displacement of the record above background at the moment of injection, as determined by extrapolation of the data to zero time, to twice the displacement of the 50 per cent standard. In the case of $\mathrm{K}^{42}$ the values used for the extrapolation were those from 1 to 2 minutes; the values obtained during the first minute were deliberately ignored.

The order of administration of the indicators was made random. In the case of the second injection, the displacement was referred to the background, including that due to the first indicator, at the moment of injection.

Hyperventilation. The effects of voluntary hyperventilation were studied in eleven subjects, who were requested to hyperventilate for 2 to 5 minutes; the measurements were performed as soon afterward as possible. Between the two isotope measurements there was an unavoidable delay of 2 minutes, during which the subjects were not hyperventilating. This, in effect, resulted in one measure- ment being made under different conditions from the other. The adverse effect of this circumstance on the estimation of the difference in the values obtained with the two indicators is obvious, but this was reduced to some extent by reversing the order of label administration from subject to subject.

Calculations. The cephalic fraction of the cardiac output was calculated as the fractional uptake of $\mathrm{I}^{131}$ extrapolated to zero time. The noncerebral cephalic fraction (uncorrected) was similarly calculated from the fractional uptake of $\mathrm{K}^{42}$. The uncorrected $\mathrm{K}^{42}$ value, it will be recalled, was obtained by extrapolation of the data obtained between 1 and 2 minutes after the administration of the label. By this time it must be presumed that that portion of the $\mathrm{K}^{42}$ which originally escaped extraction by the brain must have been recirculated and have found its place in organs other than the brain.

In animals, where the cerebral blood flow fraction is quite small, such recirculating $\mathrm{K}^{42}$ is of very little consequence. In man, where the cerebral blood flow fraction is of the order of 10 to 15 per cent of the cardiac output, it can be expected that each organ other than the brain will receive $\mathrm{K}^{42}$ in 10 to 15 per cent excess. Even such an error is not intolerable, particularly when recognized. If, however, the 10 to 15 per cent error is introduced into one of the terms of a difference it may become of real significance.

In the present experiments the $\mathrm{K}^{42}$ escaping the brain presumably found its way to the remainder of the head in proportion to its blood flow fraction. The $\mathrm{K}^{42}$ content of the head was consequently larger than its fractional flow; its subtraction from the cephalic blood flow fraction would therefore yield too small a value for the cerebral flow fraction. Fortunately, the error is easily corrected. The statements made above can be presented as two simultaneous equations as follows:

$\mathrm{I}^{131}$ uptake fraction $=$ cerebral blood flow fraction + noncerebral cephalic blood flow fraction

$\mathrm{K}^{42}$ uptake fraction $=$ noncerebral cephalic blood flow fraction $\times(1+$ cerebral blood flow fraction $)$

The solution for cerebral blood flow from these equations is :

cerebral blood flow fraction

$$
=\frac{(\mathrm{I}-1)+\left[(1-\mathrm{I})^{2}-4(\mathrm{~K}-\mathrm{I})\right]^{\frac{1}{2}}}{2}
$$

where $\mathrm{I}$ and $\mathrm{K}$ refer to the fractional uptake of these labels. This equation was used for all cerebral blood flow calculations.

\section{RESULTS}

A typical experimental record is presented in Figure 1. The subject was an Air Force physician who had been fully acquainted, through previous observation, with the procedures to be employed. $\mathrm{I}^{131}$ was given first. The rise time was 
a little more than 40 seconds and was succeeded by a virtual plateau which extrapolated to the injection time 0.9 inch above the background level. Since 50 per cent of the injected standard gives a deflection of 2.16 inches, this subject's cephalic uptake was 0.21 of the injected dose. The response after $\mathrm{K}^{42}$ showed an early peak, which subsided in a little longer than 1 minute. The remainder of the curve had a very slight slope; extrapolated with this slope to the moment of $\mathrm{K}^{42}$ injection, the displacement of the curve above baseline was 0.51 inch. With the 50 per cent standard for $\mathrm{K}^{42}$ reading 3.17 inches above baseline, this corresponded to an uptake of 8.2 per cent of the injected dose. From Equation 3 this corresponds to a cerebral blood flow fraction of 14.0 per cent of the cardiac output.

Qualitatively, all the results were similar. The values obtained for 23 normal subjects are given in Table I. Table II describes the results in 11 hyperventilating subjects.

In another set of runs, not reported here, four consecutive subjects had $\mathrm{K}^{42}$ values, equal to or greater than the $\mathrm{I}^{131}$ values. At face value, this

TABLE I

$I^{131}$ and $K^{42}$ uptake fractions of the head of normal human subjects*

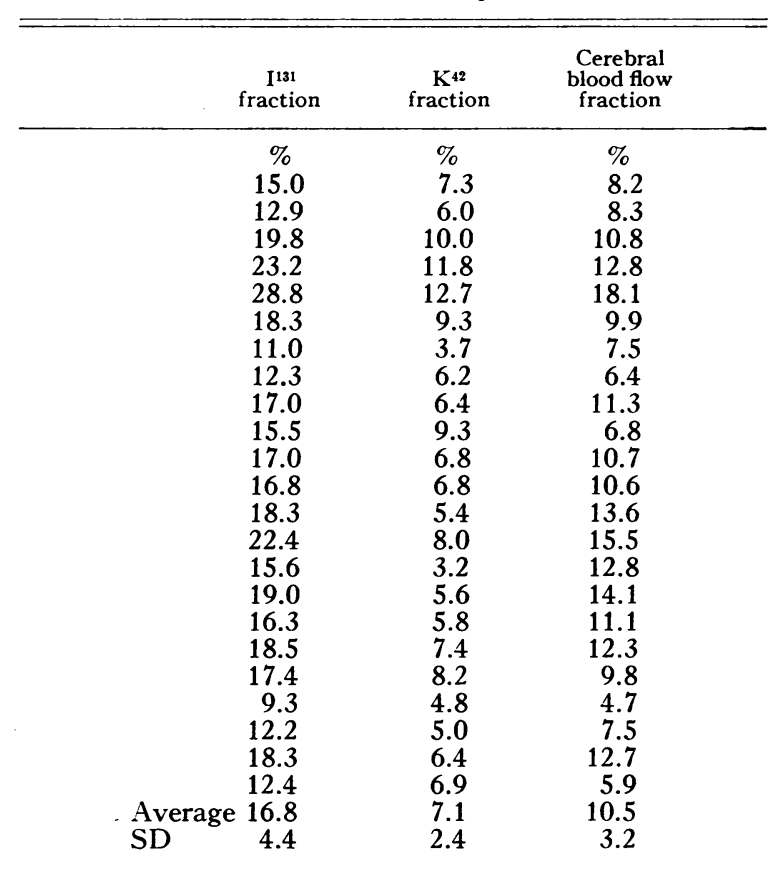

* Cerebral blood flow fraction is calculated from equation 3 of text; $I$ and $K$ values by extrapolation to zero time.
TABLE II

$I^{131}$ and $K^{42}$ uptake fractions of the head of hyperventilating human subjects *

\begin{tabular}{cccc}
\hline \hline & $\begin{array}{c}\mathrm{I}^{131} \\
\text { fraction }\end{array}$ & $\begin{array}{c}\mathrm{K}^{42} \\
\text { fraction }\end{array}$ & $\begin{array}{c}\text { Cerebral } \\
\text { blood flow } \\
\text { fraction }\end{array}$ \\
\hline$\%$ & $\%$ & $\%$ \\
14.5 & 8.3 & 6.6 \\
10.6 & 1.6 & 9.1 \\
12.0 & 7.4 & 4.9 \\
10.0 & 5.1 & 5.2 \\
8.1 & 4.5 & 3.7 \\
& 15.8 & 10.2 & 6.3 \\
& 10.9 & 7.8 & 3.3 \\
& 13.4 & 8.0 & 5.4 \\
& 12.4 & 3.0 & 9.7 \\
9.9 & 4.6 & 5.4 \\
Average & 12.4 & 7.4 & 5.4 \\
SD & 2.1 & 6.2 & 5.9 \\
& & 2.3 & 1.9 \\
\end{tabular}

* Cerebral blood flow fraction is calculated from equation 3 of text; $I$ and $K$ values by extrapolation to zero time.

implies a zero or negative cerebral blood flow value. No explanation for these anomalous results was found at the time. Subsequent consideration suggested a possible cause (see Discussion). Such values were observed in only one group of experiments.

\section{DISCUSSION}

The cephalic blood flow fraction observed in these experiments was about one-sixth of the cardiac output. This value, based on the $\mathrm{I}^{131}$ uptake, includes all of the tissues visualized by the detector.

Examination of the early portions of the curve after $\mathrm{I}^{131}$ never showed the rise and fall characteristic of an area with a small extraction ratio for the substance. Thus it appears improbable that any significant portion of the cephalic circulation passes through arteriovenous shunts or through areas that are nonexchanging with respect to this label.

After attaining its peak value, the $I^{131}$ value tended to stabilize or to show a very slight decline with time. This fact, which indicated that the tissues of the head had almost the same extraction ratio for $I^{131}$ as have those of the rest of the body, considerably simplified the extrapolation to zero time and decreased the error to be expected from a faulty identification of the zero.

Although it is possible to place considerable confidence in the value for the cephalic blood flow, 
many circumstances argue against placing similar confidence in the measurement of the noncerebral cephalic blood flow (and, by extension, the cerebral blood flow).

1) The fact that the determinations of cephalic and noncerebral cephalic blood flow fractions are not simultaneous introduces into the value obtained by subtracting one from the other any changes that have occurred between the measurements. Note that changes in cerebral blood flow, cephalic blood flow, cardiac output, or any combination of these between determinations can introduce an error into the cerebral flow fraction determined by difference.

2) If the extraction ratio of the brain for radiopotassium is not identically zero after the mass of $\mathrm{K}^{42}$ has passed through the cerebral circulation, then the "noncerebral cephalic fraction" will be increased. The cerebral fraction, derived by subtraction of this value from the cephalic fraction, will, in consequence, appear to be lower than its true value. In animal experiments the ratio between $\mathrm{K}^{42}$ and $\mathrm{I}^{131}$ uptake by brain is 6 to 7 per cent. If it is assumed that the same ratio exists in human brain, and that the cerebral blood flow fraction is 14 per cent of the cardiac output, it would suggest that approximately 1 per cent of the injected dose of $\mathrm{K}^{42}$ is located in the brain. Cephalic $\mathrm{K}^{42}$ uptake would, therefore, overestimate the noncerebral cephalic blood flow by 1 per cent of the cardiac output. The cerebral blood flow would therefore be underestimated by 1 per cent of the cardiac output.

There is the interesting possibility that the $\mathrm{K}^{42}$ uptake of neural tissue is identically zero, and that the $\mathrm{K}^{42}$ uptake by brain tissue is located in blood vessel walls, glial cells, meninges, and other connective tissues. If this is the case, the value obtained by use of Equation 3 would be more nearly representative of neural blood flow than that obtained by, for example, the nitrous oxide technique. For the present, however, it seems best to consider that the difference between the $\mathrm{I}^{131}$ and $\mathrm{K}^{42}$ values, appropriately corrected (see Equation 3), is predominantly determined by the cerebral blood flow and underestimates it to a small extent.

3) In the section on methods it was noted that $\mathrm{K}^{42}$, recirculating from brain, increased the $\mathrm{K}^{42}$ uptake of every other organ capable of acting as a reservoir for this label. The equations presented were designed to allow for recirculation of $\mathrm{K}^{42}$ from the areas visualized by the counter. No allowance was made for recirculations occurring from other areas. Such recirculations may occur through nervous tissue (cord, peripheral nerve) not included in the counter's scope, from areas with moderately low extraction ratios (aorta), and from arteriovenous shunts. Although the magnitude of shunt flow in the pentobarbitalanesthetized rat is inconsiderably small (1), its value in man in the conditions of these experiments is entirely unknown.

Clearly, the effect of extracephalic areas, which recirculate $\mathrm{K}^{42}$ and not $\mathrm{I}^{131}$, will be to increase the cephalic $\mathrm{K}^{42}$ uptake. The cerebral blood flow would therefore be underestimated. Anatomical shunts, which recirculated both labels, would increase both $\mathrm{K}^{42}$ and $\mathrm{I}^{131}$ uptake values proportionately, and thereby overestimate the cerebral fraction of the cardiac output. Despite these potential sources of error, the value obtained for the cerebral fraction of the cardiac output by the present technique is in remarkably good agreement with values obtained by other methods.

In the nitrous oxide technique the accepted value was originally approximately 14 per cent of the cardiac output. Recognition of the possibility that faulty equilibration of poorly perfused areas might influence the results upward (7) led to a revised estimate that the cerebral blood flow was approximately 10 per cent lower, i.e., about 12.5 per cent of the cardiac output. In the present experiments the value of 11 per cent of the cardiac output in the normal subjects should be revised upward 1 per cent to allow for $\mathrm{K}^{42}$ uptake by brain. The revised value of 12 per cent agrees as well as could be expected with the best estimates made by the Kety-Schmidt technique (8) in normal healthy subjects.

In further support of the validity of this method, the effects of hyperventilation can be cited. It is well known that hyperventilation reduces cerebral blood flow, as measured by the nitrous oxide technique (8), by approximately 35 per cent. About the same reduction was observed in the present experiments. Although too much emphasis should not be placed on the quantitative similarity, it is nevertheless interesting that both methods reveal a change in the same direction. 
In this connection it may be worth reconsidering the results of those experiments referred to previously, in which the $\mathrm{K}^{\mathbf{4 2}}$ fraction was greater than, or nearly equal to, the $I^{131}$ fraction. This was observed in four consecutive experiments. It seems possible that the small apparent value for the cerebral flow fraction obtained by difference in these subjects depended on changes in cerebral blood flow, noncerebral cephalic blood flow, and cardiac output occurring between the first and second measurements. It should be noted that these experiments were among the first ones done and that both experimenter and subjects were apprehensive concerning the safety of the procedure. Presumably, the first measurement $\left(\mathrm{I}^{131}\right.$ in each of these experiments) reflected more apprehension (and hyperventilation?) than the second; in any case, it seems highly probable that significant circulatory changes occurred between the measurements. This points up an important limitation of the method. It should not be employed without due caution in circumstances where circulatory conditions are changing between the two isotope measurements.

Despite the possible errors referred to above, it appears that this method for the determination of cerebral blood flow offers some unique advantages. Among them may be noted the speed and simplicity of its execution, the nontraumatic nature of the procedure, the simplicity of the instrumentation, and finally the immediacy with which the result is obtained. Granted that there are a number of potential sources of error, these factors appear almost to outweigh them, particularly when the determination of cerebral blood flow is to be made into a routine procedure.

Finally, it should be noted that considerable simplification of the procedure and the elimination of the sources of error may be accomplished by making only one measurement-that with $\mathrm{I}^{131}$ and accepting the results as indicative of the cephalic blood flow fraction. It appears possible that by shielding the head below the base of the brain pan in the manner described by Lewis and coworkers (9) it may be feasible to make this measurement without significant contribution by such structures as the teeth, tongue, thyroid, larynx, and so forth. By so doing, it may be possible to exclude from the scope of the detector all organs with significant blood supply other than the brain, and thereby to make possible the entire cerebral measurement with a single isotopic determination.

\section{SUMMARY AND CONCLUSIONS}

1) The theory of the indicator fractionation technique is presented.

2) The application of the indicator fractionation technique to the measurement of the cephalic blood flow in man (iodo ${ }^{131}$-antipyrine) and to the measurement of noncerebral cephalic blood flow $\left(\mathrm{K}^{42}\right)$ is described.

3) Results are given on the cephalic and extracerebral cephalic blood flow fractions of 23 normal and 11 hyperventilating persons. Values for cerebral blood flow fraction are calculated in these individuals. The cerebral blood flow fraction in normal persons by this technique is 11 per cent of the cardiac output; hyperventilation reduces this figure to 6 per cent.

4) Potential sources of error in the indicator fractionation technique in the cerebral blood flow measurement are described.

\section{REFERENCES}

1. Sapirstein, L. A. Regional blood flow by fractional distribution of indicators. Amer. J. Physiol. 1958, 193, 161.

2. Sapirstein, L. A. Fractionation of the cardiac output of rats with isotopic potassium. Circulat. Res. 1956, 4, 689.

3. Sapirstein, L. A., and Goodwin, R. S. Measurement of blood flow in the human hand with radioactive potassium. J. appl. Physiol. 1958, 13, 81.

4. Sapirstein, L. A., and Hanusek, G. E. Cerebral blood flow in the rat. Amer. J. Physiol. 1958, 193, 272.

5. Sapirstein, L. A., and Goldman, H. Adrenal blood flow in the albino rat. Amer. J. Physiol. 1959, 196, 159.

6. Sapirstein, L. A., and Mandel, M. J. Blood flow in the aortic wall. Circulat. Res. 1959, 7, 545.

7. Lassen, Niels A. Cerebral blood flow and oxygen consumption in man. Physiol. Rev. 1959, 39, 183.

8. Kety, S. S., and Schmidt, C. F. The effects of altered tensions of carbon dioxide and oxygen on cerebral blood flow and cerebral oxygen consumption of normal young men. J. clin. Invest. 1948, 27, 484.

9. Lewis, B. L., Sokoloff, L., Wechsler, R. L., Wentz, W. B., and Kety, S. S. A method for the continuous measurement of cerebral blood flow in man by means of radioactive krypton $\left(\mathrm{Kr}^{79}\right)$. J. clin. Invest. 1960, 39, 707. 